\title{
THE COMPLEMENTS OF LOWER CONES OF DEGREES AND THE DEGREE SPECTRA OF STRUCTURES
}

\author{
URI ANDREWS, MINGZHONG CAI, ISKANDER SH. KALIMULLIN, STEFFEN LEMPP, \\ JOSEPH S. MILLER, AND ANTONIO MONTALBÁN
}

\begin{abstract}
We study Turing degrees a for which there is a countable structure $\mathcal{A}$ whose degree spectrum is the collection $\{\mathbf{x}: \mathbf{x} \leq \mathbb{a}\}$. In particular, for degrees a from the interval $\left[\mathbf{0}^{\prime}, \mathbf{0}^{\prime \prime}\right]$, such a structure exists if $\mathbf{a}^{\prime}=\mathbf{0}^{\prime \prime}$, and there are no such structures if $\mathbf{a}^{\prime \prime}>\mathbf{0}^{\prime \prime \prime}$.
\end{abstract}

\section{INTRODUCTION}

The degree spectrum of a countable structure $\mathcal{A}$ in a finite language is the set of all Turing degrees of isomorphic copies of $\mathcal{A}$ on the universe $\omega$ :

$$
\operatorname{Sp}(\mathcal{A})=\{\operatorname{deg}(\mathcal{B}): \mathcal{A} \cong \mathcal{B} \& \operatorname{dom}(\mathcal{B})=\omega\} .
$$

Knight [7] showed that each degree spectrum is either the singleton $\{\mathbf{0}\}$ (the trivial case), or closed upward. Therefore, the degree spectrum of a non-computable structure $\mathcal{A}$ can be alternatively defined as the collection of degrees that compute copies of $\mathcal{A}$.

Slaman [12] and Wehner [13] have proved independently that there exist noncomputable structures that are computable in all non-zero degrees, i.e., the collection $\{\mathbf{x}: \mathbf{x} \not \leq \mathbf{0}\}$ is a degree spectrum of a structure. Wehner's solution used the following theorem:

Theorem 1 (Wehner [13]). There is a family $\mathcal{G}$ of sets such that $\mathcal{G}$ is uniformly $X$-c.e. if and only if $X$ is not computable.

To get a structure with the desired degree spectrum, it is enough now to use a coding $\mathcal{G} \mapsto \Gamma_{\mathcal{G}}$ of families of sets into undirected graphs such that $\mathcal{G}$ is uniformly $X$-c.e. if and only if $\Gamma_{\mathcal{G}}$ has an $X$-computable copy. For example, we can let $\Gamma_{\mathcal{G}}$ be the disconnected union of infinitely repeated graphs $\Gamma_{A}$, for each $A \in \mathcal{G}$, where each $\Gamma_{A}$ consists of a single vertex connected with independent $(n+3)$-cycles for each $n \in A$.

Note that this coding can be carried over to produce partial orderings, lattices, rings, integral domains, commutative semigroups, and 2-step nilpotent groups with the degree spectrum $\{\mathbf{x}: \mathbf{x} \not \leq \mathbf{0}\}$ (see [2]). For linear orderings [10], equivalence structures, and abelian $p$-groups [6], we only know that degree spectra can be equal to $\{\mathbf{x}: \mathbf{x} \not \leq \mathbf{0}\}$ within the $\Delta_{2}^{0}$-degrees.

Andrews's research was partially supported by NSF grant DMS-1201338. Cai's research was partially supported by an AMS Simons Travel Grant and NSF Grant DMS-1266214. Lempp's research was partially supported by AMS-Simons Foundation Collaboration Grant 209087. Kalimullin's research was partially supported by RFBR grants 15-31-20607 and 15-41-02507. Miller's research was partially supported by NSF grant DMS-1001847. Montalbán's research was partially supported a Packard Fellowship and NSF grant DMS-1363310. 
Using a coding of families into structures, Kalimullin [3, 4] generalized the result of Slaman and Wehner to the complements of lower cones of some non-zero degrees: if a degree $\mathbf{a}$ is low or c.e. then the collection $\{\mathbf{x}: \mathbf{x} \not \leq \mathbf{a}\}$ is the degree spectrum of a structure. In the next section, we will give an alternative proof for the c.e. case and, after that, we will be able to combine both cases, finding an appropriate family for a degree $\mathbf{a}$ that is low over a c.e. degree $\mathbf{g} \leq \mathbf{a}$.

Kalimullin [5] has also proved that there is a degree $\mathbf{a} \leq \mathbf{0}^{\prime \prime}$ such that the collection $\{\mathbf{x}: \mathbf{x} \not \mathbf{a}\}$ is not the degree spectrum of any structure. In the last two sections, we will see that there are large classes of degrees a with this property, and that we can even take $\mathbf{a}=\mathbf{0}^{\prime \prime}$.

\section{FAmilies that ARE UNiformly C.E. IN THE COMPLEMENT OF A CONE}

Theorem 2 (Kalimullin [4]). For each c.e. set $A$, there is a family $\mathcal{G}_{A}$ of c.e. sets such that $\mathcal{G}_{A}$ is uniformly $X$-c.e. if and only if $X \mathbb{Z}_{T} A$.

Proof (Montalbán). Without loss of generality, we may assume that the set $A \subseteq \omega$ is infinite. Fix a computable bijection $f: \omega \rightarrow A$ and let $g$ be the true stage function for $A$ defined by

$$
g(0)=0, \quad g(n+1)=(\mu s>g(n))[(\forall t>s)[f(t)>f(s)]] .
$$

Then $g \equiv_{T} A$ and the set of strings $C_{g}=\left\{\sigma \in \omega^{<\omega}: \sigma \nsubseteq g g\right.$ is c.e.

Consider the family of c.e. sets

$$
\mathcal{G}_{A}=\left\{\{n\} \oplus\left(C_{g} \cup g_{F}\right): n \in \omega \& F \text { is finite \& } F \neq W_{n}^{g}\right\},
$$

where $g_{F}=\{g \mid x: x \in F\}=\{\tau \subset g:|\tau| \in F\} \subseteq \omega^{<\omega}-C_{g}$, and

$$
\{n\} \oplus X=\{2 n\} \cup\{2 x+1: x \in X\} .
$$

The main idea of this definition is to diagonalize against all $A$-c.e. sets $W_{n}^{g}, n \in \omega$, along the co-c.e. path $g$. This ensures that the family is not $A$-c.e.; if it were $A$-c.e., then knowing $g$ we could effectively enumerate a set not equal to $W_{n}^{g}$, for each $n$, which contradicts the Recursion Theorem. On the other hand, if a string has not yet been enumerated into $C_{g}$, then it might be an initial segment of $g$, so we can use it to estimate $W_{n}^{g}$. Based on this estimation, we code a finite part of given set $X \not_{T} A$ into $F$ to eventually ensure the diagonalization $F \neq W_{n}^{g}$. We explain both parts of the proof more formally below.

First, we show that $\mathcal{G}_{A}$ is not uniformly $A$-c.e. Suppose otherwise. Since $g \equiv_{T} A$, there is a computable function $a$ such that

$$
\mathcal{G}_{A}=\left\{W_{a(k)}^{A}: k \in \omega\right\} .
$$

Define computable functions $b$ and $c$ such that, for every $n \in \omega$,

$$
2 n \in W_{a(b(n))}^{g} \text { and } W_{a(b(n))}^{g}=\{n\} \oplus W_{c(n)}^{g} \in \mathcal{G}_{A} .
$$

Now, define a computable function $d$ such that, for every $n \in \omega$,

$$
W_{d(n)}^{g}=\left\{x: g \uparrow x \in W_{c(n)}^{g}\right\} .
$$

Then $W_{d(n)}^{g} \neq W_{n}^{g}$ for every $n \in \omega$, since $W_{c(n)}^{g}=C_{g} \cup g_{F}$ with $F=W_{d(n)}^{g}$. This contradicts the Recursion Theorem, so $\mathcal{G}_{A}$ is not uniformly $A$-c.e.

Next, let $X \mathbb{Z}_{T} A$. To prove that $\mathcal{G}_{A}$ is uniformly $X$-c.e., we fix an infinite $X$ computable set $U$ that does not contain any infinite $A$-c.e. subsets. (For example, 
a set consisting of codes of initial segments of $X$ will suffice.) Let $U=\{u(0)<$ $u(1)<u(2)<\cdots\}$ for an $X$-computable function $u$. We may assume that $u(s)>s$ for every $s$.

Fix integers $n, m, s \in \omega$. Below we $X$-computably define the sequence $H_{\sigma}=$ $H_{\sigma}^{n, m, s}$ by induction on $\sigma \in \omega^{<\omega}$, beginning with the empty string $\sigma=\lambda$ :

$$
\begin{gathered}
H_{\lambda}=D_{m} ; \\
H_{\sigma * i}= \begin{cases}H_{\sigma} \cup\{u(|\sigma|)\}, & \text { if } H_{\sigma}=W_{n}^{\sigma} \text { and } s<|\sigma|, \\
H_{\sigma} & \text { otherwise, }\end{cases}
\end{gathered}
$$

where $D_{m}$ is the $m$-th finite set, and $W_{n}^{\sigma}$ is the set containing all numbers enumerated by the $W_{n}$-operator with the use at most $\sigma$ before the end of the stage $t=|\sigma|$. Let $H^{n, m, s}=\bigcup_{\sigma \subset g} H_{\sigma}^{n, m, s}$.

Suppose that $D_{m} \neq W_{n}^{g}$, and the stage $s$ is large enough that $D_{m} \neq W_{n}^{\sigma}$, for every $\sigma \subset g$ with $|\sigma|>s$. Then the definition ensures that $H^{n, m, s}=D_{m}$. Note also that for arbitrary $n, m, s \in \omega$, the set $H^{n, m, s}$ is finite, and therefore $H^{n, m, s} \neq W_{n}^{g}$; otherwise $H^{n, m, s}-D_{m}=W_{n}^{g}-D_{m}$ would be an infinite $A$-c.e. subset of $U$. Thus,

$$
\left\{H^{n, m, s}: m, s \in \omega\right\}=\left\{F: F \text { is finite } \& F \neq W_{n}^{g}\right\}
$$

for every $n \in \omega$. We also have

$$
\left\{\tau \subset g:(\exists \sigma \subseteq \tau)\left[|\tau| \in H_{\sigma}^{n, m, s}\right]\right\}=\left\{\tau \subset g:|\tau| \in H^{n, m, s}\right\}=g_{H^{n, m, s}},
$$

since $u \in H_{\sigma * i}^{n, m, s}-H_{\sigma}^{n, m, s}$ implies $u>|\sigma|$; and

$C_{g} \cup\left\{\tau:(\exists \sigma \subseteq \tau)\left[|\tau| \in H_{\sigma}^{n, m, s}\right]\right\}=C_{g} \cup\left\{\tau \subset g:(\exists \sigma \subseteq \tau)\left[|\tau| \in H_{\sigma}^{n, m, s}\right]\right\}=C_{g} \cup g_{H^{n, m, s}}$.

Therefore, the family

$$
\mathcal{G}_{A}=\left\{\{n\} \oplus\left(C_{g} \cup\left\{\tau:(\exists \sigma \subseteq \tau)\left[|\tau| \in H_{\sigma}^{n, m, s}\right]\right\}\right): n, m, s \in \omega\right\}
$$

is uniformly $X$-c.e.

Corollary 3 (Kalimullin [4]). For every c.e. degree a (in particular, for $\mathbf{a}=\mathbf{0}^{\prime}$ ), there exists a structure $\mathcal{A}$ such that $\mathbf{S p}(\mathcal{A})=\{\mathbf{x}: \mathbf{x} \not \mathbf{a}\}$.

Theorem 4. Let $G$ be a c.e. set. Then for each set $A$ with $G \leq_{T} A$ and $A^{\prime} \leq_{T} G^{\prime}$, there is a family $\mathcal{G}_{A}$ of c.e. sets such that $\mathcal{G}_{A}$ is uniformly $X$-c.e. if and only if $X \not \underline{Z}_{T} A$.

Proof. As in the previous proof, we take a function $g \equiv_{T} G$ such that the set $C_{g}=\left\{\sigma \in \omega^{<\omega}: \sigma \nsubseteq g\right\}$ is c.e.

Consider the family of c.e. sets

$$
\mathcal{G}_{A}=\left\{\{n\} \oplus\left(C_{g} \cup g_{F}\right): n \in \omega \& F \text { is finite } \& F \neq W_{n}^{A}\right\},
$$

where $g_{F}=\left\{g\lceil x: x \in F\} \subseteq \omega^{<\omega}-C_{g}\right.$.

Again, $\mathcal{G}_{A}$ is not uniformly $A$-c.e. Indeed, if $\mathcal{G}_{A}=\left\{W_{a(n)}^{A}: n \in \omega\right\}$ for a computable function $a$, then we have computable functions $b$ and $c$ such that $W_{a(b(n))}^{A}=\{n\} \oplus W_{c(n)}^{A} \in \mathcal{G}_{A}$, so that using $g \leq_{T} A$ we have a computable function $d$ such that, for all $n \in \omega$,

$$
W_{d(n)}^{A}=\left\{x: g\left\lceil x \in W_{c(n)}^{A}\right\} \neq W_{n}^{A} .\right.
$$

This contradicts the Recursion Theorem. 
Let $X \not_{T} A$. We again fix an infinite $X$-computable set $U=\{0<u(0)<u(1)<$ $u(2)<\cdots\}$ that does not contain any infinite $A$-c.e. subsets. Since $A^{\prime} \leq_{T} g^{\prime}$ there is a Turing functional $\Phi$ such that

$$
W_{n}^{A}(z)=\lim _{s} \Phi^{g}(n, z, s)
$$

for every $n, z \in \omega$. For finite uses $\sigma \in \omega^{<\omega}$, we introduce the following approximation of $W_{n}^{A}$ :

$$
V_{n}^{\sigma}=\left\{z:(\exists s<|\sigma|)\left[\Phi^{\sigma}(n, z, s) \downarrow=1 \&(\forall t<|\sigma|)\left[t>s \Longrightarrow \Phi^{\sigma}(n, z, t) \uparrow\right]\right]\right\},
$$

where $\Phi^{\sigma}(n, z, s)$ is the value (if any) computed before the stage $|\sigma|$. It is clear that $W_{n}^{A}=\lim _{\sigma \subset g} V_{n}^{\sigma}$.

The definition of the sequences $H_{\sigma}, \sigma \in \omega^{<\omega}$, is similar to one from the previous proof. In contrast with the previous proof, we have only $W_{n}^{A}=\lim _{\sigma \subset g} V_{n}^{\sigma}$ instead of $W_{n}^{g}=\bigcup_{\sigma \subset g} W_{n}^{\sigma}$. This forces us to add to the list of parameters $n, m, s \in \omega$ one more parameter $x \in \omega$ that acts as a witness of $D_{m} \neq W_{n}^{A}$. Also we need to use standard length functions $\ell_{\sigma}$ to estimate and to control the agreement between $H_{\sigma}$ and $W_{n}^{A}$.

Below is the formal inductive $X$-computable definition of the sequence $H_{\sigma}=$ $H_{\sigma}^{n, m, x, s}$, for $\sigma \in \omega^{<\omega}$ :

$$
H_{\sigma * i}= \begin{cases}H_{\lambda} \cup D_{m} ; \\ H_{\sigma} \quad & \text { if } x \notin D_{m} \triangle V_{n}^{\sigma}, x<|\sigma|, s<|\sigma|, \text { and } \\ & (\forall \rho \subset \sigma)\left[\ell_{\sigma}>\ell_{\rho}\right], \\ & \text { otherwise, }\end{cases}
$$

where $D_{m} \triangle V_{n}^{\sigma}=\left(D_{m}-V_{n}^{\sigma}\right) \cup\left(V_{n}^{\sigma}-D_{m}\right)$, and

$$
\ell_{\sigma}=\max \left\{y \leq|\sigma|:(\forall z<y)\left[z \in H_{\sigma} \Longleftrightarrow z \in V_{n}^{\sigma}\right]\right\} .
$$

Let $H^{n, m, x, s}=\bigcup_{\sigma \subset g} H_{\sigma}^{n, m, x, s}$. We must prove that

$$
\left\{H^{n, m, x, s}: m, x, s \in \omega\right\}=\left\{F: F \text { is finite } \& F \neq W_{n}^{A}\right\}
$$

for every $n \in \omega$. If $D_{m} \neq W_{n}^{A}$, then take an $x$ such that $x \in D_{m} \triangle W_{n}^{A}$. Take $s$ large enough that $x \in D_{m} \triangle V_{n}^{\sigma}$ for every $\sigma \subset g$ with $|\sigma|>s$. The definition ensures that $H^{n, m, x, s}=D_{m}$.

It remains to prove that $H^{n, m, x, s}$ is finite and $H^{n, m, x, s} \neq W_{n}^{A}$, for every $n, m, x, s$. Suppose that $H^{n, m, x, s}$ is infinite. Then $\lim _{\sigma \subset g} \ell_{\sigma}=\infty$, and so

$$
H^{n, m, x, s}=\lim _{\sigma \subset g} V_{n}^{\sigma}=W_{n}^{A}
$$

is an infinite $A$-c.e. subset of $U \cup D_{m}={ }^{*} U$, contradicting our assumption about $U$. Therefore, $H^{n, m, x, s}$ is finite.

Suppose that $H^{n, m, x, s}=W_{n}^{A}$. Then $\lim _{\sigma \subset g} \ell_{\sigma}=\infty$. Since $H^{n, m, x, s}$ is finite, by definition we should have $x \in D_{m} \triangle W_{n}^{A}=\lim _{\sigma \subset g}\left(D_{m} \triangle V_{n}^{\sigma}\right)$ and $H^{n, m, x, s}=$ $D_{m} \cup\left\{u_{1}, \ldots, u_{k}\right\}$ with $u_{i}=u\left(\left|\sigma_{i}\right|\right) \geq\left|\sigma_{i}\right|>x$ for each $i=1, \ldots, k$. Then $x \in H^{n, m, x, s} \triangle W_{n}^{A}$, which is a contradiction.

We have proved that $\left\{H^{n, m, x, s}: m, x, s \in \omega\right\}=\left\{F: F\right.$ is finite $\left.\& F \neq W_{n}^{A}\right\}$ for every $n \in \omega$. As in the previous proof, this implies that the family

$$
\mathcal{G}_{A}=\left\{\{n\} \oplus\left(C_{g} \cup\left\{\tau:(\exists \sigma \subseteq \tau)\left[|\tau| \in H_{\sigma}^{n, m, x, s}\right]\right\}\right): n, m, x, s \in \omega\right\}
$$

is uniformly $X$-c.e. 
Corollary 5. Suppose that for a degree $\mathbf{a}$ there exists a c.e. degree $\mathbf{g} \leq \mathbf{a}$ such that $\mathbf{a}^{\prime} \leq \mathbf{g}^{\prime}$. Then there exists a structure $\mathcal{A}$ such that $\mathbf{S p}(\mathcal{A})=\{\mathbf{x}: \mathbf{x} \not \mathbf{a}\}$.

Corollary 6 (Kalimullin [3]). If $\mathbf{a}^{\prime} \leq \mathbf{0}^{\prime}$, then there exists a structure $\mathcal{A}$ such that $\operatorname{Sp}(\mathcal{A})=\{\mathbf{x}: \mathbf{x} \not \leq \mathbf{a}\}$.

Corollary 7. If $\mathbf{0}^{\prime} \leq \mathbf{a}$ and $\mathbf{a}^{\prime} \leq \mathbf{0}^{\prime \prime}$, then there exists a structure $\mathcal{A}$ such that $\operatorname{Sp}(\mathcal{A})=\{\mathbf{x}: \mathbf{x} \not \leq \mathbf{a}\}$.

\section{IMPOSSIBLE DEGREE SPECTRA: A HIGHNESS PROPERTY}

A structure $\mathcal{B}$ has the c.e. extension property if each existential type $T h_{\exists}(\mathcal{B}, \vec{b})$ is c.e. As we will explain later, if the degree spectrum of $\mathcal{B}$ is the complement of a lower cone, then $\mathcal{B}$ must have this property.

Theorem 8. Let $A$ and $B$ be sets such that $B^{\prime \prime}$ has $A$-c.e. degree and $B^{\prime \prime \prime} \leq_{T} A^{\prime}$. Then each $B$-computable structure $\mathcal{B}$ with the c.e. extension property has an $A$ computable copy $\mathcal{A} \cong \mathcal{B}$.

Proof. Let $\mathcal{B}$ be a $B$-computable structure. Without loss of generality we can assume that $\mathcal{B}$ has a relational signature and the universe of $\mathcal{B}$ is $\omega$. Since $\mathcal{B}$ has the c.e. extension property, for each tuple of integers $\vec{b}$ the set $T h_{\exists}(\mathcal{B}, \vec{b})$ of all existential facts about $\vec{b}$ in $\mathcal{B}$ is c.e. Note that the relation

$$
R(\vec{b}, e) \Longleftrightarrow W_{e}=T h_{\exists}(\mathcal{B}, \vec{b})
$$

is $\Pi_{2}^{B} \subseteq \Delta_{3}^{B} \subseteq \Delta_{1}^{B^{\prime \prime}}$, so that we can fix a $B^{\prime \prime}$-computable function $e(\vec{b})$ such that

$$
W_{e(\vec{b})}=T h_{\exists}(\mathcal{B}, \vec{b}) .
$$

If $\mathcal{C}$ is a finite substructure of $\mathcal{B}$, then we will write $e(\mathcal{C})$ for $e(\vec{b})$, where $\vec{b}$ is the sequence of elements of $\mathcal{C}$ written in increasing order (as integers).

It is easy to see by a direct back-and-forth strategy that the theorem follows immediately if $e(\vec{b})$ is $A$-computable. In the general case we have $e \leq_{T} B^{\prime \prime} \leq_{T}$ $B^{\prime \prime \prime} \leq_{T} A^{\prime}$, but we also know that $B^{\prime \prime}$ has $A$-c.e. degree.

Now instead of each single back-and-forth step, we can use a straightforward technical algorithm based on the Limit Lemma:

Lemma 9. Assume that the following data is given:

(1) a finite structure $\mathcal{C}$;

(2) a monomorphism $f: \mathcal{C} \rightarrow \mathcal{B}$;

(3) a value $e(f(\mathcal{C}))$ such that $T h_{\exists}(\mathcal{B}, f(\mathcal{C}))=W_{e(f(\mathcal{C}))}$;

(4) a finite structure $\mathcal{D} \supseteq \mathcal{C}$ that is embeddable into $\mathcal{B}$ extending $f$ (we can check this fact using e $(f(\mathcal{C}))$ );

(5) an element $b \in \mathcal{B}$.

Then there is an A-computable algorithm that generates, uniformly in the given data, an infinite sequence of finite structures

$$
\mathcal{D}=\mathcal{E}_{0} \subseteq \mathcal{E}_{1} \subseteq \mathcal{E}_{2} \subseteq \cdots
$$

such that:

(1) $\mathcal{E}=\bigcup_{s} \mathcal{E}_{s}$ is finite;

(2) there is a monomorphism $g: \mathcal{E} \rightarrow \mathcal{B}$ extending $f$ such that $b \in g(\mathcal{E})$. 
Fix an $A$-c.e. set $X \equiv_{T} B^{\prime \prime}$. Our construction of an $A$-computably copy $\mathcal{A} \cong$ $\mathcal{B}$ uses the Robinson low guessing method [11], which helps tame the process of guessing facts that are computable from a low c.e. set (in this case, relative to $A$ ). To this end, we will define a Turing operator $\Gamma^{X}$. Using the Recursion Theorem and the fact that $X^{\prime} \leq_{T} A^{\prime}$, we can fix a Turing operator $\Delta$ such that $\Delta^{A^{\prime}}=\operatorname{dom} \Gamma^{X}$. We will use $\Gamma$ and $\Delta$ to certify $A$-computable guesses about computations from $X \equiv_{T} B^{\prime \prime}$.

The construction itself splits into simultaneously working modules $M_{n}, n \in \omega$; each of them produces a finite structure $\mathcal{A}_{n}$ such that $\mathcal{A}_{n} \subseteq \mathcal{A}_{n+1}$. We will let $\mathcal{A}=\bigcup_{n} \mathcal{A}_{n}$.

The module $M_{n}$ executes the procedure from Lemma 9 with the input

(1) $\mathcal{C}=\mathcal{A}_{n-1}\left(\right.$ we assume $\left.\mathcal{A}_{-1}=\emptyset\right)$;

(2) the monomorphism $f_{n-1}: \mathcal{A}_{n-1} \rightarrow \mathcal{B}$ provided by $M_{n-1}$;

(3) the value $e\left(f_{n-1}\left(\mathcal{A}_{n-1}\right)\right)$ provided by $M_{n-1}$;

(4) the finite structure $\mathcal{D} \supseteq \mathcal{C}$ equal to the union of all $\mathcal{A}_{m}, m \in \omega$, as they appear at the moment the procedure starts;

(5) the element $b=n$ from $\mathcal{B}$

The output structure $\mathcal{A}_{n}=\mathcal{E}$ should satisfy the properties:

(1) $\mathcal{A}_{n}$ is finite;

(2) there is a monomorphism $f_{n}: \mathcal{E} \rightarrow \mathcal{B}$ extending $f_{n-1}$ such that $b \in f_{n}\left(\mathcal{A}_{n}\right)$. While the procedure is executing, we are trying to calculate both a monomorphism $f_{n}: \mathcal{A}_{n} \rightarrow \mathcal{B}$ extending $f_{n-1}$ such that $b \in f_{n}\left(\mathcal{A}_{n}\right)$, and the value $e\left(f_{n}\left(\mathcal{A}_{n}\right)\right)$. Precise calculation of this data requires the $B^{\prime \prime}$-oracle, so we can pass to $M_{n+1}$ only a limit approximation based on the current $A$-computable enumeration of $X \equiv_{T} B^{\prime \prime}$. Before passing to $M_{n+1}$, we certify the current approximation: Define $\Gamma^{X}(n) \downarrow$ with the same use as the current calculation of $f_{n}$ and $e\left(f_{n}\left(\mathcal{A}_{n}\right)\right)$, then wait for $\Delta^{A^{\prime}}(n)=1$ for the current $A$-computable enumeration of $A^{\prime}$. If our approximation later becomes incorrect via inconsistency of the $\Gamma$-use, wait until we see $\Delta^{A^{\prime}}(n)=0$ and then restart the procedures in all $M_{m}$ for $m \geq n$.

Since $\Delta^{A^{\prime}}=\operatorname{dom} \Gamma^{X}$, there are only finitely many restarts for each $M_{n}, n \in$ $\omega$. Each module $M_{n}$ successfully defines a finite structure $\mathcal{A}_{n}$ with embedding $f_{n}: \mathcal{A}_{n} \rightarrow \mathcal{B}$. Since $n \in f_{n}\left(\mathcal{A}_{n}\right)$, the function $f=\bigcup_{n} f_{n}$ is an isomorphism between the $A$-computable structure $\mathcal{A}=\bigcup_{n} \mathcal{A}_{n}$ and the structure $\mathcal{B}$.

Corollary 10. If $\mathbf{a} \geq \mathbf{0}^{\prime}$ and $\mathbf{a}^{\prime} \geq \mathbf{0}^{\prime \prime \prime}$ (in particular, if $\mathbf{a}=\mathbf{0}^{(n)}$ for $n \geq 2$ ), then there is no countable structure $\mathcal{A}$ such that $\mathbf{S p}(\mathcal{A})=\{\mathbf{x}: \mathbf{x} \not \mathbf{a}\}$.

Proof. Suppose for a contradiction that $\mathbf{S p}(\mathcal{A})=\{\mathbf{x}: \mathbf{x} \not \mathbf{a}\}$. Then each existential type of $\mathcal{A}$ is c.e. relative to every element of the co-null class

$$
\left\{X \in 2^{\omega}: X \not \underline{L}_{T} \mathbf{a}\right\} \text {. }
$$

By [9], this is possible only if the existential type is c.e. Thus, $\mathcal{A}$ has the c.e. extension property. By assumption, $\mathcal{A}$ has no a-computable copy.

Consider first the case when $\mathbf{0}^{\prime \prime} \not \mathbf{a}$ a. By a modification of Friedberg's Jump Inversion Theorem (see $[8, \S 13.3$, Corollary $\mathrm{X}(\mathrm{b})]$ ), there are degrees $\mathbf{a}_{0}, \mathbf{a}_{1} \geq \mathbf{0}^{\prime}$ such that

$$
\mathbf{0}^{\prime \prime}=\mathbf{a}_{0}^{\prime}=\mathbf{a}_{1}^{\prime}=\mathbf{a}_{0} \cup \mathbf{a}_{1}
$$


Applying the same result to each of the degrees $\mathbf{a}_{0}$ and $\mathbf{a}_{1}$, we get degrees $\mathbf{b}_{0}, \mathbf{b}_{1}, \mathbf{b}_{2}$ and $\mathbf{b}_{3}$ such that

$$
\mathbf{0}^{\prime \prime}=\mathbf{b}_{0}^{\prime \prime}=\mathbf{b}_{1}^{\prime \prime}=\mathbf{b}_{2}^{\prime \prime}=\mathbf{b}_{3}^{\prime \prime}=\mathbf{b}_{0} \cup \mathbf{b}_{1} \cup \mathbf{b}_{2} \cup \mathbf{b}_{3} .
$$

Since $\mathbf{0}^{\prime \prime} \not \leq \mathbf{a}$, there exists a degree $\mathbf{b} \in\left\{\mathbf{b}_{0}, \mathbf{b}_{1}, \mathbf{b}_{2}, \mathbf{b}_{3}\right\}$ such that $\mathbf{b} \not \leq \mathbf{a}$. Then $\mathcal{A}$ has a $\mathbf{b}$-computable copy, but $\mathbf{b}^{\prime \prime}=\mathbf{0}^{\prime \prime}$ has c.e. degree relative to $\mathbf{a} \geq \mathbf{0}^{\prime}$ and $\mathbf{b}^{\prime \prime \prime}=\mathbf{0}^{\prime \prime \prime} \leq \mathbf{a}^{\prime}$. This contradicts Theorem 8 .

Suppose now that $\mathbf{0}^{\prime \prime} \leq \mathbf{a}$. Relativizing the low simple set construction, one can find an a-c.e. degree $\mathbf{c}>\mathbf{a}$ such that $\mathbf{c}^{\prime}=\mathbf{a}^{\prime}$. By a double jump inversion argument, as above, there exists a degree $\mathbf{b} \not \mathbf{a}$ such that $\mathbf{b}^{\prime \prime}=\mathbf{c}$. Then $\mathcal{A}$ has a $\mathbf{b}$-computable copy but $\mathbf{b}^{\prime \prime}=\mathbf{c}$ has $\mathbf{a}$-c.e. degree and $\mathbf{b}^{\prime \prime \prime}=\mathbf{c}^{\prime} \leq \mathbf{a}^{\prime}$. Again, this contradicts Theorem 8 .

\section{IMPOSSIBLE DEGREE SPECTRA: A NON-LOWNESS PROPERTY}

Just for convenience, we define $\mathcal{C}(\mathbf{x})$ as the class of degrees $\mathbf{a} \geq \mathbf{x}$ such that for every function $f \leq_{T} \mathbf{x}^{\prime}$, there is a $g \leq_{T}$ a that is not dominated by $f$.

Theorem 11. If $\mathbf{a} \in \mathcal{C}\left(\mathbf{0}^{\prime}\right)$, then there is no countable structure $\mathcal{A}$ such that $\operatorname{Sp}(\mathcal{A})=\{\mathbf{x}: \mathbf{x} \not \mathbf{a}\}$.

Proof. We use the following forcing lemma. The proof follows from the proof of a similar result for non- $G L_{2}$ degrees in [1, Theorem 2.8] with the observation that the function from the proof that we need to escape is computable from $\mathbf{0}^{\prime \prime}$.

Lemma 12. Let $\mathcal{P}$ be a forcing notion computable from $\mathbf{0}^{\prime}$ (i.e., both membership and the partial order are computable from $\left.\mathbf{0}^{\prime}\right)$ and let $\left\langle D_{n}\right\rangle$ be a class of dense sets such that there is a $\mathbf{0}^{\prime \prime}$-computable density function $d(p, n)=q$ which gives a forcing condition $q$ that extends $p$ and is in $D_{n}$. Then every $\mathbf{a} \in \mathcal{C}\left(\mathbf{0}^{\prime}\right)$ computes a generic sequence in $\mathcal{P}$ with respect to the dense sets $\left\langle D_{n}\right\rangle$.

We may assume that $\mathbf{a}$ is not above $\mathbf{0}^{\prime \prime}$ (because the case $\mathbf{a} \geq_{T} \mathbf{0}^{\prime \prime}$ is covered in Corollary 10). As above, we can perform double jump inversion with cone avoidance to get a degree $\mathbf{b}$ that is not below $\mathbf{a}$ and such that $\mathbf{b}^{\prime \prime}=\mathbf{0}^{\prime \prime}$.

For a contradiction, assume that $\{\mathbf{x}: \mathbf{x} \not \mathbf{a}\}$ is the degree spectrum of a countable structure $\mathcal{A}$, and let $\mathcal{M}$ be a model of $\mathcal{A}$ computable in b. As always, we assume that models are defined on $\omega$. Again, the goal is to show that a can compute a model $\mathcal{N}$ isomorphic to $\mathcal{M}$ using the fact that $\mathcal{M}$ has the c.e. extension property.

The key idea is that using $\mathbf{b}^{\prime \prime}=\mathbf{0}^{\prime \prime}$ we could compute the correct c.e. indices for the existential types of finite approximations to $\mathcal{N}$, along with associated embeddings into $\mathcal{M}$. This allows us to give a very robust construction of a structure $\mathcal{N}$ isomorphic to $\mathcal{M}$. The plan is to use this robustness and Lemma 12 to show that a can approximate such a construction well enough to also compute an isomorphic copy of $\mathcal{M}$. We need to define an appropriate forcing notion.

First, we define an auxiliary $\mathbf{0}^{\prime}$-computable ordering $\mathcal{Q}$; our forcing notion $\mathcal{P}$ will be defined based on $\mathcal{Q}$. Let $\mathcal{Q}$ be all 5 -tuples $(s, \tau, \sigma, f, e)$ where $s$ is a natural number, $\tau$ is an initial segment of the standard $\mathbf{0}^{\prime}$-computable enumeration of $\emptyset^{\prime \prime}$ at stage $s, \sigma$ is an initial segment of the model $\mathcal{N}$ we want to build, coded as a binary string, $\tau$ verifies that $f$ is a partial isomorphism from $\mathcal{N}$ to the given model $\mathcal{M}$ and that $e$ is the c.e. index of the existential type of the finitely many elements we have decided in the range of $f$. 
Given two tuples $p=(s, \tau, \sigma, f, e)$ and $q=\left(s^{\prime}, \tau^{\prime}, \sigma^{\prime}, f^{\prime}, e^{\prime}\right), q$ extends $p\left(q \leq_{\mathcal{Q}} p\right)$ if the following holds:

(1) $s \leq s^{\prime}$ and $\sigma^{\prime} \supseteq \sigma$, i.e., the model is extended consistently.

(2) either at stage $s^{\prime}$, (computable in $\mathbf{0}^{\prime}$ ) we see that the approximation to $\emptyset^{\prime \prime}$ disagrees with $\tau$ (in which case we believe $p$ is wrong); or

(a) $\sigma^{\prime}$, after coded as an existential formula over the free variables in the (assumed) type $W_{e}$, is in $W_{e}$ (so $\sigma^{\prime}$ extends $\sigma$ while respecting $W_{e}$ );

(b) $\tau^{\prime}$ verifies that $W_{e^{\prime}}$ extends $W_{e}$ in the sense that for every formula in $W_{e^{\prime}}$, after adding existential quantifiers for the last appropriate number of free variables (depending on the numbers of free variables in $W_{e}$ and $\left.W_{e^{\prime}}\right)$, is in $W_{e}$; and

(c) $f^{\prime}$ extends $f$.

(i.e., we believe $p$ is correct and we extend it to $q$ appropriately for each component.)

It is easy to see that $\mathbf{0}^{\prime \prime}=\mathbf{b}^{\prime \prime}$ computes a sequence in $\mathcal{Q}$ such that the limit of the $f$ 's is total and onto, and furthermore, such that the $\tau$ 's are always correct initial segments of $\emptyset^{\prime \prime}$. Our plan is to use the degree a to approximate such a construction. Note that in an approximation, we might figure out that some guess $p \in \mathcal{Q}$ is wrong (i.e., discover that $\emptyset^{\prime \prime}$ disagrees with $\tau$ ). In this case, we should ignore the restraints $(f, e)$ that $p$ puts on extensions, but we still want to obey the restriction placed by previous guesses that have not been proved wrong. (Note that previous guesses are not automatically respected because our order on $\mathcal{Q}$ is not actually transitive.) For this reason, we want to keep track of all guesses, so we define a forcing notion $\mathcal{P}$ by taking "chains of conditions in $\mathcal{Q}$ ".

A forcing condition in $\mathcal{P}$ is a finite sequence $\bar{p}$ of conditions $\left\langle p_{0}, p_{1}, \ldots, p_{n}\right\rangle$ such that each $p_{i}$ is in $\mathcal{Q}$ and $p_{i}$ extends $p_{j}$ whenever $i>j$. Given two conditions $\bar{p}$ and $\bar{q}$, we say $\bar{q}$ extends $\bar{p}$ if $\bar{p}$ is an initial segment of $\bar{q}$ (as sequences). It is easy to see that the forcing notion $\mathcal{P}$ is computable from $\mathbf{0}^{\prime}$.

Consider the dense sets

$$
D_{n}=\left\{\bar{p}: \text { in the last condition } p_{m} \text { of } \bar{p}, \tau_{m} \text { is a correct initial segment of } \emptyset^{\prime \prime}\right.
$$
and $n$ is in both the domain and range of $f_{m}$.

We show that $\mathbf{0}^{\prime \prime}$ computes a density function $f$ such that $f(\bar{p}, n)$ extends a given $\bar{p}$ and is in $D_{n}$. First of all, given $\bar{p}=\left\langle p_{0}, p_{1}, \ldots, p_{m}\right\rangle, \mathbf{0}^{\prime \prime}$ can figure out whether the $\tau$ part of these conditions are correct or not, and find a stage $s_{0}$ when we detect all incorrect ones in the $\mathbf{0}^{\prime}$-computable enumeration. Now for the last remaining correct $p_{i}, \sigma_{m}$ is, by definition of the partial order, in $W_{e_{i}}$. This means that the current model can still be consistently mapped to the given model $\mathcal{M}$ by a partial isomorphism extending the correct $f_{i}$ in $p_{i}$. So we can extend the sequence by adding a new condition $(s, \tau, \sigma, f, e)$ where $\sigma$ contains $n$ in the universe; $f$ is an extension of the partial isomorphism as above by adding $n$ into both the domain and range; $e$ is the correct existential type of the current elements in the domain (and range) of $f$; all is witnessed by a correct initial segment $\tau$ of $\emptyset^{\prime \prime}$; and $s$ is a stage number greater than $s_{0}$ and large enough to enumerate $\tau$ in the $\mathbf{0}^{\prime}$-computable enumeration.

Now applying Lemma 12, we get a generic sequence $G$ computable in a meeting all the dense sets $D_{n}$ 's. Let $\left(p_{0}, p_{1}, p_{2}, \ldots\right)$ be the sequence of $\mathcal{Q}$-conditions in $\bar{p} \in G$. This gives an a-computable model $\mathcal{N}$ by projecting the elements of $G$ 
onto their $\sigma$ coordinates, i.e., $\mathcal{N}=\cup_{i} \sigma_{i}$. In addition, when we meet a dense set $D_{n}$, we guarantee that the partial isomorphism $f$ is correct and preserved in the construction by any forcing extension, and we make sure that $n$ is in the domain and range of $f$. So the union of these correct partial isomorphisms is an isomorphism from $\mathcal{N}$ to $\mathcal{M}$. This gives the desired contradiction.

As a final remark, recall that a degree $\mathbf{a} \geq \mathbf{0}^{\prime}$ is $\overline{G L_{2}}$ over $\mathbf{0}^{\prime}$ if $\mathbf{a}^{\prime \prime}>\left(\mathbf{a} \vee \mathbf{0}^{\prime \prime}\right)^{\prime}$. It is well-known that all degrees $\overline{G L_{2}}$ over $\mathbf{0}^{\prime}$ are in the class $\mathcal{C}\left(\mathbf{0}^{\prime}\right)$, and restricted to the degrees in $\left[\mathbf{0}^{\prime}, \mathbf{0}^{\prime \prime}\right]$ they are exactly the degrees that are non-low ${ }_{2}$ over $\mathbf{0}^{\prime}$, i.e., $\mathbf{a}^{\prime \prime}>\mathbf{0}^{\prime \prime \prime}$. Recall that if a degree $\mathbf{d}$ is low over $\mathbf{0}^{\prime}$, then $\{\mathbf{x}: \mathbf{x} \not \mathbf{a}\}$ is a degree spectrum, so in the interval $\left[\mathbf{0}^{\prime}, \mathbf{0}^{\prime \prime}\right]$, we only have a very small gap left, namely the degrees that are low $_{2}$ but not low over $\mathbf{0}^{\prime}$.

Corollary 13. If $\mathbf{0}^{\prime} \leq \mathbf{a} \leq \mathbf{0}^{\prime \prime}$ and $\mathbf{a}^{\prime \prime}>\mathbf{0}^{\prime \prime \prime}$, then there is no countable structure $\mathcal{A}$ such that $\mathbf{S p}(\mathcal{A})=\{\mathbf{x}: \mathbf{x} \not \leq \mathbf{a}\}$.

Remark 14. It follows from Theorem 8 that every low $_{2}$ algebraic structure with the c.e. extension property has copies in the degrees $\mathbf{a}$ such that $\mathbf{a} \geq \mathbf{0}^{\prime}$ and $\mathbf{a}^{\prime} \geq \mathbf{0}^{\prime \prime \prime}$, i.e., in the degrees of functions dominating all $\mathbf{0}^{\prime}$-computable functions. Moreover, by the proof of Theorem 11, every low 2 algebraic structure with the c.e. extension property has copies in the degrees $\mathbf{a} \in \mathcal{C}\left(\mathbf{0}^{\prime}\right)$, i.e., in the degrees $\mathbf{a} \geq \mathbf{0}^{\prime}$ such that there is no $\mathbf{0}^{\prime}$-computable function dominating all $\mathbf{a}$-computable functions. Note also that by Corollary 3, not every low algebraic structure with the c.e. extension property has a copy in a c.e. degree $\mathbf{a}<\mathbf{0}^{\prime}$, even if $\mathbf{a}^{\prime}=\mathbf{0}^{\prime \prime}$.

\section{REFERENCES}

[1] Minzhong Cai and Richard Shore. Domination, forcing, array nonrecursiveness and relative recursive enumerability. J. Symbolic Logic. 77:33-48, 2012.

[2] Denis R. Hirschfeldt, Bakhadyr Khoussainov, Richard A. Shore and Arkadii M. Slinko. Degree spectra and computable dimensions in algebraic structures. Annals of Pure and Applied Logic. 115 (1-3):71-113, 2002.

[3] Iskander Sh. Kalimullin. Spectra of degrees of some structures. Algebra Logika, 46(6):729-744, 2007.

[4] Iskander Sh. Kalimullin. Almost computably enumerable families of sets. Sb. Math., 199(10):1451-1458, 2008.

[5] Iskander Sh. Kalimullin. Restrictions on the degree spectra of algebraic structures. Siberian Math., 49(6):1034-1043, 2008.

[6] Iskander Kalimullin, Bakhadyr Khoussainov and Alexander Melnikov. Limitwise monotonic sequences and degree spectra of structures. Proc. Amer. Math. Soc. 141:3275-3289, 2013.

[7] Julia F. Knight. Degrees coded in jumps of orderings. J. Symbolic Logic, 51(4):1034-1042, 1986.

[8] Rogers, H. Jr., Theory of recursive functions and effective computability, McGraw Hill, New York, 1968.

[9] Karel de Leeuw, Edward F. Moore, Claude E. Shannon, and Norman Shapiro. Computability by probabilistic machines, Automata studies, pp. 183212. Annals of mathematics studies, no. 34. Princeton University Press, Princeton, N. J., 1956.

[10] Russell Miller. The $\Delta_{2}^{0}$-spectrum of a linear order. J. of Symbolic Logic. 66 (2):470 - 486, 2001.

[11] Robert W. Robinson. Interpolation and embedding in the recursively enumerable degrees. Ann. of Math., 93:285-314, 1971.

[12] Theodore A. Slaman. Relative to any nonrecursive set. Proc. Amer. Math. Soc., 126(7):2117$2122,1998$.

[13] Stephan Wehner. Enumerations, countable structures and Turing degrees. Proc. Amer. Math. Soc., 126(7):2131-2139, 1998. 
(Andrews, Lempp, Miller) Department of Mathematics, University of Wisconsin, 480 Lincoln Dr., MADison, WI 53706, USA

E-mail address: andrews@math.wisc.edu, lempp@math.wisc.edu, jmiller@math.wisc.edu

$U R L$ : www.math.wisc.edu/ andrews

$U R L$ : www. math.wisc.edu/ lempp

$U R L$ : www.math.wisc.edu/ jmiller

(Cai) Department of Mathematics, Dartmouth College, Hanover, NH 03755, USA

E-mail address: mingzhong.cai@dartmouth.edu

$U R L$ : math.dartmouth.edu/ cai

(Kalimullin) Institute of Mathematics and Mechanics, Kazan Federal University, Kremlevskaya ST. 18, 420008 KazAN, RUSSIA

E-mail address: Iskander.Kalimullin@kpfu.ru

URL: kpfu.ru/main?p_id=10721

(Montalbán) Department of Mathematics, University of California-Berkeley, Evans Hall \#3840, Berkeley, CA 94720, USA

E-mail address: antonio@math.berkeley.edu

$U R L$ : www. math. berkeley.edu/ antonio 